\title{
El acto médico en distorsión: relación sin médico y sin paciente: desplazamiento del poder de decisión al conjunto familia-medios de comunicación-estado clientelar
}

\author{
Claudia Muravchik, Hospital Dr. Julio C. Perrando, Argentina
}

\begin{abstract}
Resumen: La relación médico-paciente ha ido evolucionando a lo largo del tiempo: desde la hegemonía médica al empoderamiento del paciente; y desde el binomio mencionado a la incorporación de otros involucrados (equipo interdisciplinario-familiaamigos-asesores legales). Deberíamos referirnos (más aún cuando el caso involucra pacientes pediátricos o incapaces) a la relación equipo tratante- familia y su contexto social. Se describen dos casos que resultan paradigmáticos para ilustrar lo que sería un desplazamiento del eje gravitacional de poder dentro de esta relación y la aparición de una nueva hegemonía. Esta hegemonía estaría representada por la familia que, a través de relaciones de poder establecidas con políticos y con los medios de comunicación y construidas sobre la enfermedad del paciente, decide sobre las medidas terapéuticas a implementar basándose en sus propias prioridades. Esto redunda en una serie de beneficios para los familiares, sin que resulte en beneficios reales para el paciente y que, en casos extremos como los referidos, resulta en encarnizamiento terapéutico.
\end{abstract}

Palabras clave: bioética, encarnizamiento terapéutico, relación médico-paciente, estado clientelar

\begin{abstract}
The relation doctor-patient has been developing as time went by: since the medical hegemony to the empowerish of the patient; and since the mentioned binomial to the incorporation of others involved(Interdisciplinary team, family, friends, legal councellors). We shoud refer (even more when the case involves pediatric patients or incapables) to the relation treating team family and its social context. There are 2cases described that result paradigmatic to illustrate what would be a displacement of the gravitacional axis of power inside this relation and the appearance of a new hegemony. This hegemony would be represented for the family that throughout power relations established with politicians and media and built over the illness of the patient decides over the therapeutic measures to implement based in its own priorities. This results into a series of benefits for the relatives, without being profitable in real benefits for the patient and that in extreme cases such as the ones mentioned, it results in therapeutic cruelty.
\end{abstract}

Keywords: Bioethics, Therapeutic Cruelty, Relation Doctor-Patient, Clientelism

\section{Introducción}

$\mathrm{L}$ a relación médico-paciente, díada constitutiva del llamado acto médico y más antigua que la medicina misma, puede definirse como una modalidad de relación interpersonal que supone el encuentro de dos individualidades. Como todo lo que atañe a la persona, este encuentro no es atemporal ni universal: sucede en un contexto socio-histórico particular, que le otorga significación a la enfermedad que los lleva al encuentro y también los significa a ellos, en sus roles de médico y del paciente.

Esta relación intersubjetiva surge en la búsqueda del bienestar por parte del paciente y se desarrolla en el terreno de la comunicación, entendida ésta como una experiencia antropológica. A través del lenguaje se despliega este juego de poderes entre quien busca ayuda y quien tiene la capacidad de brindarla. Y evoluciona.

Evoluciona en relación al lugar donde se desarrolla: Inicialmente en la intimidad de la habitación del paciente, luego en el consultorio y finalmente en los grandes hospitales.

Evoluciona en cuanto a los actores que lo protagonizan, alejándonos cada vez más de la mínima dupla médico- paciente: acompañando al médico se suman profesionales y técnicos que participan en la 
atención de la salud (otros médicos especialistas interconsultores, enfermeros, kinesiólogos, extraccionistas, técnicos de laboratorio, etc.). Acompañando el paciente está presente la familia y se suman otros personajes que influyen en mayor o menor medida en la toma de decisiones, como por ejemplo amigos, asesores legales y la comunidad en general a la que pertenece. Ya no se trata de la "relación médicopaciente" sino de una "relación equipo de salud- usuarios".

Evoluciona, finalmente, la gravitación del poder dentro de la relación que se desplaza sobre los diferentes actores, dando lugar a la descripción de diferentes paradigmas: del paradigma médico hegemónico, paternalista, al proceso de emancipación de los pacientes que desemboca en un paradigma horizontal de decisiones compartidas, con un eje gravitacional puesto en la autonomía del paciente.

A fin de analizar y reflexionar sobre la continua evolución del acto médico, se presenta el reporte de dos casos que pueden resultar paradigmáticos para ilustrar lo que sería un desplazamiento del eje gravitacional de poder dentro de esta relación y la aparición de una nueva hegemonía.

\section{Caso 1}

Paciente femenina de 14 años de edad a su ingreso al Servicio de Terapia Intensiva, con diagnóstico de Lipofuscinosis Neuronal Cerúlea, enfermedad neurológica degenerativa progresiva que comenzó a manifestarse a sus cuatro años de edad. Al momento de presentar la complicación (neumonía) que la lleva a la internación, presentaba un cuadro de estado vegetativo persistente, postración, ceguera, sordera, miopatía generalizada y alimentación por sonda nasogástrica. Su familia estaba compuesta por su madre conviviente, compañera y cuidadora diaria, y su padre no conviviente desde el diagnóstico. Obrero él, sostén económico de la paciente, en su frustración por no conseguir financiación para tratarla, inicia una lucha mediática para conseguir que reciba tratamiento de sostén y pueda acceder a un tratamiento experimental (de eficacia no comprobada y alto costo) con implante de células madre. Logra una ley nacional y realiza campañas de recolección de fondos, lo cual le otorga un lugar destacado en su comunidad y relaciones a nivel político nacional. Concreta el implante, y previo a un segundo tratamiento se complica. Ingresa a UTI, pese a las objeciones de los médicos y con la orden de "no debe faltarle nada" de autoridades gubernamentales. La madre, a diferencia del padre que veía excelente evolución en su hija, reconocía el carácter terminal de la evolución de la enfermedad, y refería que todas las intervenciones le provocaban dolor y sufrimiento sin ver mejoría alguna. Pero el padre imponía sus deseos en las decisiones y se negaba a cualquier posibilidad de suspensión de medidas extraordinarias. La paciente permaneció internada durante 2 años, multiagredida y atravesando múltiples agonías. Fallece en UTI, luego de una agonía prolongada, con la prensa presente y las autoridades gubernamentales amenazando a los médicos por haber sugerido la suspensión de medidas terapéuticas y la posibilidad de optar por la implementación de "muerte digna".

\section{Caso 2}

Paciente de sexo femenino, nace en el seno de una familia indigente, última de 5 hermanos. Luego de un embarazo complicado, sucede el parto (con alta sospecha de mala praxis) y nace prematura extrema, sufre hipotermia: evoluciona con una lesión neurológica severa (menos del 10\% de encéfalo funcionante). El caso toma estado público en forma casi inmediata desnudando graves falencias de la Salud Pública; las autoridades políticas de turno, conscientes del efecto mediático del caso se presentan a exigir al equipo de salud interviniente "una atención de excelencia" y la exigencia de prolongar la sobrevida de la paciente a como dé lugar. En este contexto, la familia por decisión del Estado, recibe trabajo, vivienda, motovehículos y teléfonos. Permanentemente rodeada de políticos y periodistas transcurre su lenta recuperación. Se plantea la limitación del esfuerzo terapéutico y la muerte digna, que es expuesta por la prensa como una sentencia de muerte médica. Los padres se separan de inmediato y la madre continúa con la tenencia de la niña, que retorna al hogar en estado vegetativo persistente y asistencia de cuidadores domiciliarios costeados por el Estado, en espera de un viaje a China para experimentar un trasplante de células madres. 


\section{Discusión}

Se analizan los casos desde una perspectiva humanística: El acto médico utilizando el cristal de la bioética.

Los casos presentados se sitúan para su análisis en torno a las decisiones al final de la vida en pacientes incapaces: las pacientes se enfrentan a enfermedades complejas, progresivas, profundamente discapacitantes, en estado terminal, con necesidad de tratamientos cruentos, invasivos y costosos que de ser exitosos, sólo prolongarían la sobrevida dependientes de apoyos vitales mecánicos, sin cambios en la calidad de vida o en el pronóstico terminal, dicho de otra manera: retardarían el momento de la muerte. ¿Quiénes son los responsables de velar por los “mejores intereses" de estas pacientes (entendido esto como protegerlo de un sufrimiento innecesario o prolongar una vida de experiencias muy limitadas)?

Se suele considerar a los padres como los representantes naturales de estos mejores intereses (si las pacientes estuvieran o hubiesen estado dotadas de autonomía, se solicitaría su opinión o se trabajaría con la familia respecto de sus directivas anticipadas en caso de que las hubieran manifestado). En ambos casos los padres exigen medidas extraordinarias, que se podrían encuadrar en lo que se conoce como futilidad terapéutica o encarnizamiento terapéutico.

En discusiones bioéticas, incluso también en estudios referidos a la comunicación y la salud, con frecuencia son los médicos identificados como responsables del encarnizamiento terapéutico, "éste suele ser el resultado de un equipo de salud formado en la medicina biologista, de los temores de estos a los conflictos judiciales o a conflictos con los familiares (la llamada "medicina defensiva") y de la habitual adhesión al supuesto de "defender la vida" sobre todas las cosas. La particularidad en los casos expuestos reside en que la iniciativa de mantener medidas terapéuticas fútiles proviene de los mismos padres, que incluso se niegan a escuchar a los médicos tratantes, y activan de inmediato lo que ellos significan como sus mecanismos de "control": los medios de comunicación y las autoridades o políticos de turno.

¿Es válido analizar el encarnizamiento terapéutico surgido de la propia familia?

La primer pregunta que surge en torno al cuestionamiento anterior es ila familia resulta siempre la representante natural de los mejores intereses del paciente? No, no siempre resulta de ese modo. A veces intereses egoístas de los padres pueden impedirles ver el sufrimiento en la agonía y se niegan a permitir el devenir de la muerte. No se trata de poner en tela de juicio el amor de los padres, pero también es cierto que en nombre del amor se han cometido atrocidades.

Pero entonces: ¿son estos padres excepciones, desalmados que no comprenden la futilidad del tratamiento en la terminalidad? Si extendemos el análisis fuera del núcleo familiar aislado y lo contextualizamos, podemos incluir la idea de éxito terapéutico que ofrece la sociedad de hoy en las expectativas de estos padres. Como consecuencia de la medicalización de la cultura, los usuarios tienen expectativas desmedidas respecto a la medicina, el fracaso no es una opción; y esto se suma a la fascinación por terapéuticas novedosas, de poca o ninguna evidencia de efectividad. La sociedad, y los padres con ella, ven éxito en el paradigma actual del enfermo como "Hombre ciborg", quien vence a la muerte aliado con la tecnología, mitad hombre mitad máquina.

Podemos ampliar entonces el análisis a lo sociopolítico, llevemos de la intimidad a estas relaciones paciente-familia al contexto en el que se desarrollan: las pacientes referidas pertenecen a familias pobres de la Argentina, son personas excluidas del sistema en una sociedad neoliberal. A partir del suceso enfermedad, la invisibilidad social termina y se inicia un proceso de inclusión: existen para una sociedad que hasta el minuto previo les daba la espalda. El Estado aparece ante el reclamo mediático de los padres, toma posición y valida este encarnizamiento, ignora a las pacientes y centra las prioridades en los deseos de los padres. ¿Por qué? Actuando como dueños de la salud, respondiendo al modelo de "Estado de Bienestar" producto de su alianza con la Medicina que como ciencia se alía al poder político y económico y transforma la Salud en una mercancía: cambian publicidad por apoyo gubernamental. Me parece muy representativo citar a Alejandro Navas García, en su texto "El imaginario social de la Salud en la Cultura Moderna" donde dice: "Desde el momento en que el Estado moderno, omnipotente regulador de todos los ámbitos de la vida, incluye la 
salud entre las prestaciones que ofrece a sus ciudadanos, la mentalidad así inducida y la lógica electoral imponen un crecimiento continuo de nuevas demandas. Pronto será imposible financiar tanto servicio, pero el gobierno que se atreva a plantear claramente las dimensiones de la crisis y recorte - o racionalice, por emplear el término técnico y eufemístico - el gasto, se expondrá a perder las siguientes elecciones".

Y los medios de comunicación, constructores de la realidad en esta sociedad mediatizada sobrevuelan la escena, sin imparcialidad, buscando sensacionalismo o al servicio del poder, condenan también a la oscuridad al equipo de salud y a las pacientes.

¿Cómo repercute esta realidad en la actitud del médico y del equipo de salud? ¿Cuál resulta ser el rol que le toca interpretar en esta relación particular? El médico desde el punto de vista ético y legal debe respetar la autonomía, en este caso ejercida por los padres: puede alegar objeción de conciencia pero no puede abandonar al paciente. Puede objetar las publicaciones y los dichos en los medios de comunicación pero sólo a través de la vía legal, con tiempos muy distintos a los de los sucesos y sufriendo un desgaste personal y económico.

Llegamos entonces a la pregunta: ¿Dónde están el médico y su paciente, sino rehenes de la situación? La relación se desarrolla sin médico y sin paciente.

\section{Conclusiones}

Los casos presentados representan en forma extremas las características actuales de la relación equipo de salud-usuarios, que en mayor o menor medida se replican en el ejercicio diario de las profesiones de la salud.

Un viejo adagio dice referido a la función del médico: "cuando no cura, alivia, y cuando no, consuela". Y es el acto médico, este encuentro entre dos personas, donde deben encontrar médico y paciente las circunstancias más propicias para establecer una comunicación efectiva que permita al enfermo pedir ayuda en su enfermedad (sea lo que para él signifiquen) y al médico poder ejercer su arte y aplicar los elementos que la ciencia médica pone a su alcance en beneficio del paciente, en respuesta a sus necesidades particulares.

En los casos analizados, esta comunicación resulta imposible, al quedar distanciados por las circunstancias familiares, culturales y sociopolíticas particulares. El paciente y el médico son silenciados, y cabe entonces la pregunta: ¿Existe el acto médico en estas circunstancias?

El acto médico se distorsiona y se resume a un servicio técnico sin criterios propios, el acto médico como mercancía y convertido en una prestación deshumanizada: resulta en uno más de los beneficios sociales en manos del Estado, manejado por los políticos y el pueblo como un derecho o bien reclamable y negociable dentro del clientelismo político.

Humanizar al acto médico es revalorizarlo como un encuentro entre un médico y paciente personas, y como tal único en sus particularidades. La participación del entorno familiar, social y político no debe suplantar, sino acompañar y facilitar la comunicación real y efectiva en este encuentro irrepetible, para que el resultado sea valioso para el paciente, el médico y la sociedad a la que pertenecen. 


\section{REFERENCIAS}

Alonso, G. V. (2007). Acerca del clientelismo y la política social: reflexiones en torno al caso argentino. Revista del CLAD, 37, pp. 1-10.

Araya Umaña, S. (2002). Las representaciones sociales: ejes teóricos para su discusión. Cuadernos de Ciencias Sociales, 127. Costa Rica: FLACSO.

Boer, M. (2008). Planteos éticos de la práctica de la medicina en pacientes pediátricos con pronóstico reservado. Sus familiares y la búsqueda de alternativas terapéuticas. Rev. Hosp. Italiano B. Aires, 28(2), pp. 95-100.

Burbinsky, B. \& Naser, M. (1999). Reflexiones acerca de la Relación Médico-Paciente. Arch. Arg de Pediatría, 97(1), pp. 43.

Cambra, U. C., Hevia, T. M., \& Iturrizaga, A. U. (coord.). (2011). Comunicación y salud: nuevos escenarios y tendencias. Madrid: Editorial Complutense.

Cannellotto, A. \& Luchtenberg, E. (comp.). (2008).Medicalización y sociedad. Lecturas críticas sobre un fenómeno en expansión. Argentina: Observatorio Argentino de Drogas (SEDRONAR)

Cruz-Hernández. M. (2004). Un renovado reto en la formación pediátrica: la relación médicopaciente familia. Educación Médica, 7(4), pp. 119-12.

De Lillo, L. (2011). Del encarnizamiento terapéutico a una muerte digna. Revista de Bioética y Socioantropología en Medicina, VI(1). Disponible en: http://www.humanidadesmedicas.sld.cu/index.php/hm/article/view/33/56.

Feo, Ó. (2008). Las políticas neoliberales y su impacto sobre la formación en salud pública. Medicina Social, 3(4), pp. 275-284.

Lázaro, J. \& Gracia, D. (2006). La relación médico-enfermo a través de la historia. An. Sist. Sanit. Navar., 29(3), pp. 7-17.

Martínez, O. C., Marchante, M. C. F., Morales, I. G., \& Martínez, D. P. S. (2010). La relación médico paciente en la actualidad y el valor del método clínico. Medisur, 8(5), pp. 110-120.

Mcnab, M.E. \& Beca, J.P. (2010). ¿Existen límites en la decisión de los padres sobre el tratamiento de los hijos? Rev Chil Pediatr., 81(6), pp. 536-540.

Moreno, C. \& Rovira, C. (2009). Imaginarios: Desarrollo y aplicaciones de un concepto crecientemente utilizado en las Ciencias Sociales. Investigación para la Política Pública, Desarrollo Humano, HD-08-2009, RBLAC-UNDP, New York

Ocampo-Martínez, J. (2002). La bioética y la relación médico-paciente. Cir Ciruj, 70 (EneroMarzo), pp. 55-59.

Rodríguez Arce, M.A. (2008). Relación Médico-Paciente. La Habana: Editorial Ciencias Médicas.

\section{SOBRE LA AUTORA}

Claudia Muravchick: Médica cirujana, especialista universitaria en Terapia Intensiva. Médica de staff de UTI, actualmente médico staff de Unidad de Epidemiología. Antecedentes: Jefa de Sala Terapia Intermedia, Directora del Servicio de Medicina Prehospitalaria, Directora de Hospital, Coordinadora Hospitalaria de CUCAI Chaco (Organismo dedicado a procuración y transplante de órganos). Posgrados: Bioética, Gestión hospitalaria, Vulnerabilidad y catástrofe, Enseñanza de Ciencias, Medicina Antropológica. 